\title{
A SCALING ANALYSIS OF THE EFFECTS OF NATURAL CONVECTION, WHEN SAL'NIKOV'S REACTION: $P \rightarrow$ A $\rightarrow$ B OCCURS, TOGETHER WITH DIFFUSION AND HEAT TRANSFER IN A BATCH REACTOR
}

\author{
A.N. CAMPBELL, S.S.S. CARDOSO AND A.N. HAYHURST* \\ Department of Chemical Engineering, University of Cambridge, Pembroke Street, Cambridge, CB2 3RA \\ * Correspondence to: Professor A.N. Hayhurst, Department of Chemical Engineering, University of \\ Cambridge, Pembroke Street, Cambridge CB2 3RA, U.K. \\ E-mail: anh1000@hermes.cam.ac.uk
}

Sal'nikov's chemical reaction in its simplest form consists of two consecutive first-order steps, producing a product $\mathrm{B}$ from a precursor $\mathrm{P}$ via an active intermediate $\mathrm{A}$, in $\mathrm{P} \rightarrow \mathrm{A} \rightarrow \mathrm{B}$. The first step is assumed here to be thermoneutral, with zero activation energy, whilst the second step is exothermic and has a positive activation energy. These properties make this mechanism one of the simplest to display thermokinetic oscillations, as seen in e.g. cool flames or a batch reactor. We first consider a pure gas, $\mathrm{P}$, undergoing Sal'nikov's reaction in a closed spherical vessel, whose walls are held at a constant temperature. Natural convection becomes significant once the temperature is high enough for the Rayleigh number $(\mathrm{Ra})$ to reach $\sim 10^{3}$. The subsequent behaviour of the system depends on the interaction between convection, diffusion of heat and mass, and chemical kinetics. By examining the governing equations, we develop and evaluate scales for the characteristic velocity, the concentration of the intermediate $\mathrm{A}$ and the temperature rise during the progress of the reaction, for the two extreme cases when transport is dominated, in turn, by diffusion and then by natural convection. These scales depend on the characteristic timescales for the interacting phenomena of chemical reaction, diffusion and natural convection. Typically, the characteristic velocity in a relatively small reactor of radius $0.27 \mathrm{~m}$ is as large as $0.3 \mathrm{~m} \mathrm{~s}^{-1}$, when the temperature rise is $\approx 100 \mathrm{~K}$ near the centre of the vessel. Our theoretical predictions are verified by full numerical simulations. Oscillations of both the temperature and the concentration of the intermediate, A, are considered; the accompanying flow field proves to be toroidal, with the fluid ascending close to the reactor's axis, but descending adjacent to its walls. In addition, the effects of such process variables as the initial 
temperature of the batch reactor and its contents, the pressure and also the size of the reactor are all assessed, together with a consideration of what happens when the reaction proceeds in the liquid phase. In this case, because of the different physical properties of a liquid and a gas, natural convection is more intense than in the gas-phase and is quite likely to lead to turbulence and good mixing.

Key words: batch reaction, cool flames, natural convection, oscillations, Sal'nikov, scaling, oscillating reactions

\section{INTRODUCTION}

Ryszard Pohorecki has been a most welcome visitor to the Department of Chemical Engineering in Cambridge over many years. Indeed, it is true to say that, as a result of Danckwerts's hospitality, Ryszard has a second home at Pembroke College, Cambridge. Thus it is no surprise that Ryszard is often seen proudly sporting his Pembroke College tie, whilst he moves in his friendly and diplomatic way as a spokesman for European Chemical Engineering. Two of us (S.S.S.C. and A.N.C.) are members of Pembroke College, but all three authors heartily salute Ryszard on the occasion of his seventieth birthday and sincerely wish him "Many Happy Returns", particularly to Cambridge. We choose as our theme a topic not far from some of his work - a previously neglected consideration of an exothermic reaction proceeding batchwise in a fluid, so that the release of heat generates natural convection in the reacting mixture. It turns out that the resulting convection may be sufficiently strong to be turbulent. In any event, natural convection of the fluid is likely to augment the usual processes of heat and mass transfer, all of them topics at the very heart of Chemical Engineering.

Natural convection can be induced in a fluid by an exothermic reaction once the temperature has risen sufficiently that the Rayleigh number, $\mathrm{Ra}=\left(\beta g L^{3} \Delta T\right) /(\kappa v)$, reaches $\sim 10^{3}$ (Tyler, 1966; Turner, 1979). The interaction of the exothermic chemical reaction, diffusion of both heat and matter, and natural convection determine the behaviour of such a system in e.g. a closed batch reactor. In this paper, we consider the effect of natural convection on the reaction proposed by Sal'nikov (1949), occurring first of all in the gas-phase in a closed spherical vessel. The reaction consists of two consecutive first-order steps:

$$
\mathrm{P} \stackrel{1}{\longrightarrow} \mathrm{A} \stackrel{2}{\longrightarrow} \mathrm{B}
$$

where a precursor $\mathrm{P}$ is converted to a product $\mathrm{B}$ via an active intermediate $\mathrm{A}$. Reaction (I) is the simplest to display thermokinetic oscillations, such as characterise many more complex reaction schemes $(e . g$. cool 
flames or oscillations in a reactor). The first step is assumed to be thermoneutral, with $E_{l}$, its activation energy, and $q_{1}$, the exothermicity of step 1, both equal to zero. Step 2 is considered to be exothermic, with $E_{2}>0$ and $q_{2}>0$. Reaction (I) has been extensively studied in the well-mixed limit (Gray and Scott, 1990a), where the effects of diffusion can be neglected. Work has also been carried out on the other limiting case, where the transport of heat and mass is purely diffusive (Gray and Scott, 1990b). This diffusive limit corresponds to reaction occurring in microgravity, as studied experimentally by Pearlman (2000) and numerically by Fairlie and Griffiths (2001, 2002) and Fairlie et al. (2005). More recently, numerical studies have investigated the influence of natural convection on Sal'nikov's reaction. Thus Cardoso et al. (2004a) reported some preliminary results on the development of natural convection in a spherical vessel containing a gas undergoing Sal'nikov's reaction. The development of convection was considered in greater detail by Cardoso et al. (2004b), who proposed the regime diagram shown in Figure 1 to describe the behaviour of the system. The axes of this diagram correspond to ratios of characteristic timescales $(\tau)$ for the three interacting phenomena in the system, namely chemical reaction, diffusion and convection, as discussed below. Also presented by Cardoso et al. (2004b) was an initial scaling analysis of the system. The present work improves that analysis. Several other aspects have been covered by Campbell et al. (2005a, b).

\section{THEORY}

The equation for $a$, the concentration of the active intermediate $\mathrm{A}$, is

$$
\frac{\partial a}{\partial t}+\underline{u} . \nabla a=D_{A} \nabla^{2} a+k_{1} p_{0} \exp \left(-k_{1} t\right)-k_{2} a,
$$

where $p_{0}$ is the initial concentration of $\mathrm{P}$. It is assumed in this equation that the concentration of $\mathrm{P}$ in the reactor is initially uniform, and that it remains so, equal to $p_{0} \exp \left(-k_{l} t\right)$, throughout the course of the reaction. This assumption depends on $k_{l}$ being independent of temperature (because $E_{l}=0$ ) and holds only for relatively small increases in temperature. The conservation of energy is described by

$$
\frac{C_{V}}{C_{P}} \frac{\partial T}{\partial t}+\underline{u} . \nabla T=\kappa \nabla^{2} T+\frac{q_{2} k_{2}}{\rho_{0} C_{P}} a,
$$

where $\rho_{0}$ is the density at the initial temperature $T_{0}$. The familiar Navier-Stokes equations describe the conservation of momentum

$$
\frac{\partial \underline{u}}{\partial t}+\underline{u} . \nabla \underline{u}=-\frac{1}{\rho_{0}} \nabla\left(\mathscr{P}-\mathscr{P}_{0}\right)+v \nabla^{2} \underline{u}+\frac{\rho-\rho_{0}}{\rho_{0}} \underline{g},
$$

where $\mathscr{P}_{0}$ is the initial pressure in the reactor. The conventional Boussinesq approximation is adopted, i.e. it is assumed that the density only varies in the buoyancy term of the Navier-Stokes equations. In this term the density varies as $\rho=\rho_{0}\left[1-\beta\left(T-T_{0}\right)\right]$, where $\beta$ is the coefficient of thermal expansion. The final 
equation required is the continuity equation. The adoption of the Boussinesq approximation allows the continuity equation to be written in its incompressible form, i.e.

$$
\nabla \cdot \underline{u}=0
$$

Initially the gas is pure $\mathrm{P}$ at a temperature $T_{0}$, and is motionless. The wall is held at $T_{0}$ throughout, and the familiar no-slip condition applies. There is also assumed to be no flux of any species at the wall, where no heterogeneous reactions occur. However, there is of course heat transfer to the wall.

\section{SCALING ANALYSIS}

In order to make equations (1) - (4) dimensionless, the following seven dimensionless variables were defined:

$$
a^{\prime}=\frac{a}{a_{0}} ; p^{\prime}=\frac{p}{p_{0}} ; T^{\prime}=\frac{T-T_{0}}{\Delta T} ; \underline{u^{\prime}}=\frac{\underline{u}}{U} ; \mathscr{P}^{\prime}=\frac{\mathcal{P}-\mathscr{P}_{0}}{\rho_{0} U^{2}} ; \underline{x^{\prime}}=\frac{\underline{x}}{L} \text { and } t^{\prime}=\frac{U t}{L}, \quad(5 \mathrm{a}-\mathrm{g})
$$

where $a_{0}$ is a characteristic concentration of species A, $\Delta T$ is the characteristic temperature rise and $U$ is the characteristic velocity. At this stage, these three scales are unknown, whereas $p_{0}$ and $L$ (the radius of the reactor) are defined for a given system. Using these scales, equations (1) - (4) become, respectively:

$$
\begin{gathered}
\frac{\partial a^{\prime}}{\partial t^{\prime}}+\underline{u^{\prime}} \cdot \nabla^{\prime} a^{\prime}=\frac{D_{A}}{U L} \nabla^{\prime 2} a^{\prime}+\frac{k_{1} p_{0} L}{U a_{0}} p^{\prime}-\frac{k_{2,0} L}{U} \exp \left(\frac{\phi T^{\prime}}{1+\eta T^{\prime}}\right) a^{\prime}, \\
\frac{C_{V}}{C_{P}} \frac{\partial T^{\prime}}{\partial t^{\prime}}+\underline{u^{\prime}} \cdot \nabla^{\prime} T^{\prime}=\frac{\kappa}{U L} \nabla^{\prime 2} T^{\prime}+\frac{q_{2} k_{2,0} a_{0} L}{\rho_{0} U C_{P} \Delta T} \exp \left(\frac{\phi T^{\prime}}{1+\eta T^{\prime}}\right) a^{\prime}, \\
\frac{\partial \underline{u}^{\prime}}{\partial t^{\prime}}+\underline{u^{\prime}} \cdot \nabla^{\prime} \underline{u^{\prime}}=-\nabla^{\prime} \mathcal{P}^{\prime}+\frac{v}{U L} \nabla^{\prime 2} \underline{u^{\prime}}-\frac{L \beta \Delta T T^{\prime}}{U^{2}} \underline{g}, \\
\nabla^{\prime} \cdot \underline{u^{\prime}}=0,
\end{gathered}
$$

where $k_{2,0}$ is $k_{2}$ evaluated at the wall's temperature, $T_{0}$, and

$$
\eta=\frac{\Delta T}{T_{0}} \text { and } \phi=\frac{E_{2} \Delta T}{R T_{0}^{2}} .
$$

It is also useful at this stage to define the five characteristic timescales:

$$
\tau_{\text {step } 1}=\frac{1}{k_{1}} ; \tau_{\text {step } 2}=\frac{1}{k_{2,0}} ; \tau_{\text {diffusion } H}=\frac{L^{2}}{\kappa} ; \tau_{\text {diffusionA }}=\frac{L^{2}}{D_{A}} \text { and } \tau_{\text {convection }}=\frac{L}{U},
$$

for the various interacting phenomena in the system, namely the two steps of reaction (I), diffusion of both heat and the intermediate A and finally convection. The relative values of these timescales will determine the behaviour of the system. In fact, it can be shown (Campbell et al. 2005b) that if the initial temperature, $T_{0}$, is fixed, together with the variables: $C_{p}, C_{v}, D_{A}, \kappa, v, k_{1}, A, E_{2}$ and $q_{2}$, the behaviour of the system is totally defined by the three dimensionless groups: 


$$
p^{\prime}=\frac{p}{p_{0}} ; \quad \frac{\tau_{\text {step } 2}}{\tau_{\text {diffusion }}}=\frac{\kappa}{k_{2,0} L^{2}} ; \quad \frac{\tau_{\text {step } 2}}{\tau_{\text {convection }}}=\frac{U}{k_{2,0} L}
$$

Thus Figure 1 gas been drawn for a constant value of $p^{\prime}$. Of course, transport can be controlled by either diffusion or convection, and the form of the unknown scales will depend on which mechanism dominates. We examine each region, in turn, in order to determine the most appropriate scales in each case. It is important to note that in Figure 1, a straight line through the origin is one of constant Ra; in fact, the slope equals $1 /(\operatorname{RaPr})^{1 / 2}$, with $\operatorname{Pr} \sim 1$ for a gas, but $\operatorname{Pr}=6.6$ for liquid water at $22^{\circ} \mathrm{C}$.

\section{Transport Controlled by Diffusion}

For Rayleigh numbers less than a threshold value (of $\approx 10^{3}$ ) (Tyler, 1966; Turner, 1979), natural convection will be unimportant, so that diffusion will be the dominant mechanism for the transfer of mass. Likewise, heat transfer is by thermal conduction, i.e. the diffusion of heat. When diffusion dominates transport, the temperature and concentration fields are approximately spherically symmetric, with the maximum temperature occurring close to the centre of the reactor. In this case, the characteristic velocity, $U$, is given by $D / L$, where $D$ is either the thermal or the molecular diffusivity. We firstly assume that equation (6) for species $\mathrm{A}$ is dominated by the kinetic terms. This yields a scale for the characteristic concentration of species A:

$$
a_{0} \sim \frac{k_{1}}{k_{2,0}} p_{0}=\frac{\tau_{\text {step } 2}}{\tau_{\text {step } 1}} p_{0},
$$

i.e. the steady state hypothesis. If we similarly assume that the diffusion and generation terms dominate in the energy balance (7), we can derive a scale for $\Delta T$ as:

$$
\Delta T \sim \frac{q_{2} k_{1} L^{2}}{C_{P} \kappa}=\frac{\Delta T_{a d}}{\gamma} \frac{\tau_{\text {diffusion } H}}{\tau_{\text {step } 1}},
$$

where $\Delta T_{a d}$ is the adiabatic temperature increase $\left(=q_{2} / C_{V}\right)$ and $\gamma$ is the ratio of specific heats $\left(C_{P} / C_{V}\right)$. This scaling assumes that $\tau_{\text {convection }} \gg \tau_{\text {diffusion }} \gg \tau_{\text {step } 2}$.

\section{Transport Controlled by Convection}

When the Rayleigh number becomes sufficiently large, natural convection becomes the dominant transport mechanism. Thus for $10^{3}<\mathrm{Ra}<10^{6}$, the convective flow is expected (Turner, 1979) to be laminar. Natural convection distorts the spherical symmetry observed when diffusion dominates transport; it also leads to the formation of a hot zone above the centre of the reactor (Cardoso et al., 2004a, b). If we assume that the convection and buoyancy terms dominate in the Navier-Stokes equations (8), we can define an appropriate scale for the characteristic velocity as 


$$
U \sim[\beta g L(\Delta T)]^{1 / 2} .
$$

Similarly, if we assume that the kinetic terms dominate equation (6) (as in the previous section) and that convection and generation dominate the thermal balance (7), we can define a scale for $\Delta T$ as:

$$
\Delta T \sim\left(\frac{q_{2}}{C_{P}}\right)^{2 / 3}\left(\frac{k_{1}^{2} L}{\beta g}\right)^{1 / 3}=\frac{\Delta T_{a d}}{\gamma} \frac{\tau_{\text {convection }}}{\tau_{\text {step } 1}} .
$$

This scaling for $\Delta T$ assumes that $\tau_{\text {diffusion }} \gg \tau_{\text {convection }} \gg \tau_{\text {step } 2}$. Interestingly, application of this condition (by assuming that convection and the generation of A dominate equation (6)) leads to:

$$
a_{0} \sim \frac{k_{1} p_{0} L}{U}=\frac{\tau_{\text {convection }}}{\tau_{\text {step } 1}} p_{0}
$$

In the next section we compare these scales to the results of a full numerical solution of all the governing equations, for the two separate cases where either diffusion or natural convection is the dominant mode of transport.

It should be noted that the regime diagram in Figure 1 is specifically for Sal'nikov's kinetic scheme (I). If a more complex mechanism were considered, the diagram would change by acquiring an extra dimension for each new rate coefficient. However, the criterion, that for the onset of convection in a sphere: $\mathrm{Ra}>\sim 10^{3}$, is independent of the reaction mechanism. Likewise, the criterion that $\mathrm{Ra}>\sim 10^{6}$ for a transition from laminar to turbulent flow is also independent of the chemistry. Of course, the particular conditions at which these transitions occur, e.g. the temperature and velocity distributions in a given vessel, will depend on the detailed kinetics, as suggested by equations (13) - (15).

\section{NUMERICAL SOLUTION}

Equations (1) - (4) were solved numerically for a spherical batch reactor with a fixed wall temperature, $T_{0}$, containing initially pure gas $\mathrm{P}$, which then undergoes Sal'nikov's reaction (I). The equations were solved using Fastflo (Fastflo Tutorial Guide, 2000), which is a PDE solver utilising the finite element method. The algorithm used was the same as that outlined by Cardoso et al. (2004b).

For the purpose of the numerical simulations, we consider the thermal decomposition of di-t-butyl peroxide in a spherical reactor. This reaction was chosen because it can be shown to behave like Sal'nikov's reaction under certain conditions (Griffiths et al., 1988; Gray and Griffiths, 1989); thus experimental studies using a semi-batch reactor with the slow admission of reactant mimics the effect of step 1 in Sal'nikov's reaction (I). Such an arrangement is suitable for investigating Sal'nikov's reaction in the well-mixed limit; however, it is not suitable for cases which are not spatially uniform. Sal'nikov's reaction has been studied numerically by Fairlie and Griffiths (2002) in both the well-mixed and zerogravity extremes, as well as by Cardoso et al. (2004a, b) when natural convection is important. The following constants were chosen to match those used by Cardoso et al. (2004a, b). The temperature of the 
wall of the spherical reactor, $T_{0}$, was held constant at $500 \mathrm{~K}$ and the physicochemical properties used were as follows: the initial molar density $\rho_{0}=8.2 \mathrm{~mol} \mathrm{~m}^{-3}$ (corresponding to a pressure of 0.34 bar at $500 \mathrm{~K}$ ), the heat capacity at constant volume $C_{V}=190 \mathrm{~J} \mathrm{~mol}^{-1} \mathrm{~K}^{-1}$, and the exothermicity of step $2, q_{2}=400 \mathrm{~kJ}$ $\mathrm{mol}^{-1}$. We define the base case chemistry such that the rate constant $k_{l}=0.025 \mathrm{~s}^{-1}$, corresponding to $\tau_{\text {step } 1}$ $=40 \mathrm{~s}$, and $k_{2}=Z_{2} \exp \left(-E_{2} / R T\right)$ with $Z_{2}=2 \times 10^{15} \mathrm{~s}^{-1}$ and $E_{2} / R=18280 \mathrm{~K}$. These values give $k_{2,0}=0.265 \mathrm{~s}^{-1}$, and hence $\tau_{\text {step } 2}=3.77 \mathrm{~s}$, which is thus approximately an order of magnitude faster than step 1. Furthermore, the simplifying assumption that the Lewis and Prandtl numbers are unity was made. This implies that $v=\kappa=D_{A}$, i.e. the diffusivities of momentum, heat and chemical species were considered to be equal.

Computations were done for a range of reactor sizes at several values of $\mathrm{Ra}$, both in the region where diffusion controls transport and when convection dominates. To further verify the scales developed, the kinetic constants were also varied. Given that the scales for $\Delta T$ (equations (13) and (15)) depend on $k_{l}$ only, a range of values for $k_{l}$ was considered. The pre-exponential factor in $k_{2}$ was halved to confirm that $\Delta T$ is indeed independent of $k_{2}$.

\section{NUMERICAL RESULTS}

\section{Diffusive Regime}

We begin by considering the cases when diffusion controls transport, i.e. the cases $\mathrm{Ra}=0$ and $\mathrm{Ra} \sim$ 600. This means that as far as Figure 1 is concerned, we are only considering systems on, or very near, the vertical axis (so $g=0$ or close to that value), where only diffusion and reaction occur. Another example of Ra being low is a gaseous system at a low pressure; in this context it is noted below that $\mathrm{Ra}$ is proportional to the square of the pressure. Nevertheless, it turns out that there are three identifiable regions, where the system behaves differently, as shown more clearly in Figure 2(a-c). The temporal development of the temperature and the concentration of $\mathrm{A}$ at the centre of the reactor for three different

values of $\tau_{\text {diffusion }}$ are plotted. In the three cases presented, the chemistry is that of the base case, i.e. $\tau_{\text {step } 1}$ $=40 \mathrm{~s}$ and $\tau_{\text {step } 2}=3.77 \mathrm{~s}$ at $500 \mathrm{~K}$, and $\tau_{\text {diffusion }}$ has been varied by changing the radius, $L$, of the reactor (in this case the graphs represent $L=10,20$ and $30 \mathrm{~mm}$, respectively). For a small reactor $(L<0.01 \mathrm{~m})$, the working point is on the vertical axis of Figure 1, far away from the origin. For this case, Figure 2(a) shows slow growths and decays of both the temperature and the concentration of A with time. In addition, there is only a relatively small increase in temperature (of $\sim 8 \mathrm{~K}$ ) to the maximum at the centre of the reactor, so the system behaves almost isothermally. For these cases with a small $\tau_{\text {diffusion, }}$, we would expect the temperature and concentration fields to be in effect uniform, with the exception of the thermal and concentration boundary layers at the wall. This approximate spatial uniformity decreases the magnitude of the convection and diffusion terms in equation (6) relative to that of the reaction terms. Therefore, we 
expect $\Delta T$ to have the form of equation (13), i.e. there is a dependence on $k_{l}$, but not $k_{2}$. Indeed, the concentration and temperature fields obtained numerically were virtually uniform in these cases. The temperature and concentration only change by $\sim 1 \%$, on moving from the wall to the centre of the reactor. This small variation in temperature and concentration leads us to describing the system as approximately spatially uniform. Additionally our numerical results show that the decay in temperature and concentration (as shown by Figure 2(a)) is proportional to $\exp \left(-k_{1} t\right)$, thus lending support to the hypothesis that $k_{l}$ is the dominant kinetic parameter in this system. In all three cases plotted in Figure $2(a-c)$, the fields of temperature and of the concentration of the intermediate A were spherically symmetric.

When we increase the size of the reactor, we move into a region of instability, where the temperature and the concentration of A exhibit temporal oscillations, as shown in Figure 2(b) for the centre of the reactor. In fact, the concentration of A oscillates in anti-phase with the temperature, as has been shown previously (Cardoso et al. 2004a, b). It was found that oscillations only occurred for values of the reactor's radius, $L$, in a narrow band, whose location depended on the physical and kinetic parameters used. It seems that (see below), when diffusion is the dominant transport mechanism, the range of $L$, over which oscillatory behaviour is observed, corresponds to the region where the characteristic timescales for diffusion and reaction in step 2 are of similar magnitude i.e. $\tau_{\text {diffusion }} \approx \tau_{\text {step } 2 \text {. }}$

For $L>0.03 \mathrm{~m}$, the working point moves along the vertical axis of Figure 1 closer to the origin. Now the temporal evolutions of temperature and the concentration of $\mathrm{A}$ at the centre of the reactor are shown by Figure 2(c). Instead of the oscillations, there is now an initial peak in the concentration curve, which then rapidly decays to almost zero. The temperature now rises by $\sim 100 \mathrm{~K}$, because of heat removal from a larger vessel being slower. The plot in Figure 2(c) shows an initially fast rise in temperature and then there is a distinct 'kink' in the curve (at $\sim 2 \mathrm{~s}$ ), when the concentration of the intermediate reaches a steady value, close to zero. The 'kink' in the temperature curve can be explained by examining equation (2), i.e. the energy conservation equation. When the concentration of A falls rapidly to virtually zero, the heat generation term in equation (2) effectively disappears. It is this swift change in the form of the governing equation that causes the observed change in the temporal development of the temperature.

The plot in Figure 3 is a check of equation (13) and shows the computed values of $\gamma\left(\Delta T / \Delta T_{a d}\right)$ plotted against $\tau_{\text {diffusion }} / \tau_{\text {step } ~}$ for when diffusion is the dominant transport mechanism. The temperature rise was taken to be that at the centre of the reactor, because this is where $\Delta T$ is at its maximum value, due to the spherical symmetry. Figure 3 evidently reveals three regimes. There is linearity for high and very low $\tau_{\text {diffusion }} / \tau_{\text {step } 1}$. The region between them is where oscillations occur. Equation (13) suggests that there should be a linear plot in Figure 3. Therefore our scale, equation (13), for $\Delta \mathrm{T}$ is of the correct form. 
When $\tau_{\text {diffusion }} / \tau_{\text {step I }}>0.1$, equation (13) can be modified by adding a constant factor, which can be found from Figure 3 by the least squares method. Thus the characteristic temperature rise is given by:

$$
\begin{gathered}
\gamma \frac{\Delta T}{\Delta T_{a d}}=(0.141 \pm 0.002) \frac{\tau_{\text {difffusion }}}{\tau_{\text {step } 1}},\left(\frac{\tau_{\text {difffusion }}}{\tau_{\text {step } 1}}<0.04\right) ; \text { and } \\
\gamma \frac{\Delta T}{\Delta T_{a d}}=(0.100 \pm 0.003) \frac{\tau_{\text {difffusion }}}{\tau_{\text {step } 1}}+(0.030 \pm 0.002),\left(\frac{\tau_{\text {diffusion }}}{\tau_{\text {step } 1}}>0.1\right) .
\end{gathered}
$$

In the narrow region between these two linear regimes, where oscillations occur, the 'error bars' in Figure 3 show the range of the oscillations, measured from the first peak to the first trough). Oscillations were only observed in this narrow band, where $\tau_{\text {diffusion }} \sim \tau_{\text {step } 2}$.

\section{Convective Regime}

The behaviour of the system when convection is more important than diffusional transport was investigated by again examining full numerical solutions, but for $\mathrm{Ra} \sim 5000$ and 21500 . The convective flow in both these cases should be laminar. The flow-field is such that the gas rises vertically along the axis of symmetry and falls downwards close to the cooler walls, thus forming a toroidal vortex. Figure 4a plots the streamlines computed for the flow induced by natural convection. In outline, the system behaves as follows. The walls of the reactor are kept at a constant temperature. Whilst reaction proceeds, heat is released and consequently the temperature of the gas rises. Because the temperature of the gas rises above that of the walls, heat is removed from the system at the walls. This coupling of heat generation and loss causes a hot zone to form at the centre of the reactor. This in turn results in a gravitationally unstable density distribution in the top section of the reactor and so leads to the development of the familiar Rayleigh-Bénard convection (Turner, 1979). Figure 4(b) plots the temperature and density along the vertical axis of the reactor. The hot gas near the centre of the reactor rises quickly initially and moves into the hottest part of the reactor (in the top half). However, it slows as it passes through the hot zone, due to the decreased density difference. The hot gas then contacts the relatively cold walls, where it cools and descends relatively rapidly due to the large density differential. In the lower half of the reactor the density distribution is intrinsically stable, with the flow being induced by the descending, cooler gas at the wall. This downward flow of cool gas results in a relatively slow upward flow (around the centreline of the reactor) of gas displaced from the bottom of the reactor. Whilst this gas rises, it heats up and hence accelerates. The situation in Figure 4 refers not just to Sal'nikov's mechanism, but to any exothermic reaction proceeding in a similar vessel.

As mentioned previously, the spherical symmetry of the temperature and concentration fields seen in the diffusive regime is disrupted by the convective flow. Because of the 'hot zone' above the centre of the reactor (Cardoso et al., 2004a, b), we examine below, the temperature rise, $\Delta T$, at a point $L / 2$ above 
the centre of the reactor (i.e. a point three quarters of the way up the vertical axis), instead of at the centre, to give a better indication of the maximum value of $\Delta T$ within the reactor. Oscillations were observed over a much wider range of values than in the diffusive regime, and in fact, virtually every case studied when convection was significant exhibited oscillations.

Figure 5 shows a plot of the computed maximum vertical velocity at the centre of the reactor against $\left(g L^{2}\right)^{1 / 3}$, which arises from substituting equation (15) into (14). The 'error bars' show the range of the observed oscillations in the velocity. The linear form of this plot indicates that our scale, equation (14), is of the correct form. It is interesting to note that for a $10 \mathrm{~cm}$ diameter vessel undergoing a reaction in terrestrial conditions (i.e. $g=9.81 \mathrm{~m} \mathrm{~s}^{-2}$ ), velocities of $\sim 10 \mathrm{~cm} \mathrm{~s}^{-1}$ were observed. Velocities of up to 30 $\mathrm{cm} \mathrm{s}^{-1}$ were computed for similarly sized vessels for larger values of $g\left(=30 \mathrm{~m} \mathrm{~s}^{-2}\right)$. The characteristic velocity, $U$, can therefore be expressed as:

$$
U=(0.31 \pm 0.02)[\beta g L(\Delta T)]^{1 / 2} .
$$

This equation contains $\Delta T$. To check its magnitude, Figure 6 shows $\gamma\left(\Delta T / \Delta T_{a d}\right)$ plotted against $\tau_{\text {convection }} / \tau_{\text {step } 1}$; as predicted by equation (15), there is a clear linear relationship. In fact, the characteristic temperature rise when convection dominates transport can be expressed as:

$$
\gamma \frac{\Delta T}{\Delta T_{a d}}=(4.00 \pm 0.08) \frac{\tau_{\text {convection }}}{\tau_{\text {step } 1}} .
$$

Thus our numerical simulations have confirmed the form of the scales developed above, when diffusion and convection are, respectively, the dominant transport mechanism. These scales allow the general behaviour of any given system to be predicted. It should be noted, however, that these scales were developed for a system where $\tau_{\text {step } 1}$ is the dominant kinetic timescale. Thus, the behaviour may well change if $\tau_{\text {step } 1}$ and $\tau_{\text {step } 2}$ are of similar order.

\section{Oscillations}

The oscillations of $a$ and $T$ in the above systems result from the interaction between chemical kinetics and heat transfer. This truth is not just restricted to Sal'nikov's reaction (Gray and Scott, 1990), so consideration of Sal'nikov's system indicates how more complex mechanisms might behave. Previous work in the well-mixed region (e.g. Gray and Scott, 1990) has shown that oscillations in the temperature and the concentration of the intermediate A occur in anti-phase. The physical basis of these anti-phase oscillations can be easily explained. The reactor initially contains pure precursor $\mathrm{P}$, with no intermediate. When reaction (I) proceeds, the intermediate, A, is produced in step 1 and so begins to accumulate. Step 2 of reaction (I) consumes A, but generates heat, because of its exothermicity. The temperature within the system therefore rises. Because step 2 of the reaction has an Arrhenius temperature dependence, it is selfaccelerating. Once the temperature is such that the depletion of A in step 2 exceeds the production of $\mathrm{A}$ in 
step 1, the concentration of A begins to decline. The rate of heat generation, however, is still greater than the rate of heat loss to the walls; consequently the temperature continues to increase. The concentration of A decreases to a point where the rate of heat generation in step 2 is less than the rate of heat loss, causing the temperature to decrease. With the temperature falling, the rate of depletion of A in step 2 also drops, until once again the rate of production of $\mathrm{A}$ is greater than the rate of its destruction in step 2 . At this point the cycle begins once again. The observed oscillations are damped (see Figure 2(a)), because the precursor, $\mathrm{P}$, is continually consumed during the course of the reaction, so the production of A follows an exponential decay arising from the kinetics of step 1 . This oscillatory behaviour is due to the interaction of the highly non-linear thermal feedback, due to the Arrhenius temperature dependence of step 2, the timescales of steps 1 and 2 of reaction (I) and the nature of heat transfer from the reactor. Interestingly, oscillations with $T$ and $a$ in phase can occur; they are discussed elsewhere by Campbell et al. (2005b). The entire region of Figure 1 where oscillations occur has been found by performing very many simulations and is shown approximately in Figure 7, which is discussed below.

\section{The Effect of Varying Process Parameters}

Examination of the form of the scales developed in the previous sections, along with the expression for the Rayleigh number allows us to predict how the system will respond to variations in certain process parameters. In particular, it is important to reveal the effects of pressure, reactor size and the phase of the system (i.e. gas or liquid) on the intensity of convection in the reactor. Let us first consider the effect of increasing the pressure in a gas-phase reaction. The kinetic theory of gases indicates that $v$ and $\kappa$, the momentum and thermal diffusivities, are both inversely proportional to pressure. This means that the Rayleigh number is proportional to $\mathscr{P}^{2}$. Thus, increasing the pressure increases the Rayleigh number and therefore the intensity of the convective flow. A system represented by a point on the regime diagram of Figure 7 actually moves vertically downwards, when the pressure is increased, because the terms in the abscissa are independent of pressure. The system could accordingly move from a situation of negligible convection at low pressure, through one of laminar flow, to eventually turbulent convection, when the pressure is increased.

The effect of increasing the reactor's size is shown by the solid curved line in Figure 7. (n.b. $\left.\tau_{\text {step } 2} / \tau_{\text {diffusion }} \propto 1 / L^{2} ; \tau_{\text {step } 2} / \tau_{\text {convection }} \propto 1 / L^{1 / 3}\right)$. It is clear from this plot that making the reactor smaller significantly reduces the Rayleigh number, thereby making diffusive processes more significant. Figure 7 shows that when small reactors are considered, even relatively small increases in reactor size can significantly alter the Rayleigh number, which ex hypothesi has an explicit dependence on $L^{3}$. There is also a dependence of $\Delta T$ on $L$ via equation (13) or (15). 
As for the effect of temperature on Ra, for a purely gaseous system in the convective regime, $\mathrm{Ra}$ turns out to be inversely proportional to $T_{0}^{11 / 3}$, according to the simplest version of the kinetic theory of gases. Otherwise, the main effect of temperature on the location of the working point in Figures 1 and 7 is via the exponential in the Arrhenius function for $k_{2}$. Consequently any increase in temperature reduces $\tau_{\text {step } 2}$ and so moves the working point towards the origin of Figures 1 and 7, whose abscissa and ordinate are both proportional to $\tau_{\text {step } 2}$.

The final effect highlighted on Figure 7 is the effect of moving from a reaction in the gas-phase to one in the liquid-phase. Comparing the relative magnitudes of the terms in the Rayleigh number for typical gases and liquids indicates that Rayleigh numbers will be at least an order of magnitude higher for liquid-phase systems, for similar increases, $\Delta T$, in temperature and identical values of $L$. Thus comparing Ra for reactions with the same $\Delta T, g$ and $L$ in water and air at normal temperature and pressure indicates that $\mathrm{Ra}$ with water is some 240 times $\mathrm{Ra}$ with air. In fact, $\Delta T$ for a reaction in the liquid-phase is likely to be only $\sim 10 \%$ larger than for a reaction with the same kinetic parameters $\left(q_{2}, k_{1}, k_{2}, \ldots\right)$ in the gasphase. The overall result is that gaseous and liquid systems occupy quite different areas of Figures 1 and 7. In a liquid-phase reaction, natural convection is therefore likely to be more vigorous and important than in a gas-phase reaction with similar kinetic parameters. Certainly Figure 7 indicates that natural convection is likely to be most important (i.e. turbulent) in reactors on an industrial scale, because of the dependence on $L^{3}$. Whenever natural convection is stronger, Figure 7 makes it clear that there is a greater likelihood of oscillations in the reactor. This is because of the quite different portions of the two axes (of Figure 7) occupied by the region wherein oscillations occur. Thus on the vertical axis, the region for oscillations roughly extends over $0.85<\tau_{\text {step } 2} / \tau_{\text {diffusion }}<2$, whereas along the horizontal axis, oscillations occur over approximately $10<\tau_{\text {step } 2} / \tau_{\text {convection }}<70$, i.e. a change by a factor of 7 .

\section{CONCLUSION}

Scales have been developed for the characteristic concentration of intermediate A, temperature rise and velocity when Sal'nikov's reaction occurs in a closed spherical vessel, both for the case where diffusion is the dominant transport mechanism, and when convection dominates. In both cases the characteristic concentration of the intermediate A was determined purely by the kinetics and the temperature rise was shown to be proportional to the ratio of the characteristic timescales for the dominant transport mechanism and the rate-controlling step of the reaction. It should be noted that this behaviour may differ if the relative magnitudes of the kinetic parameters are significantly altered. Using these scales, along with order of magnitude arguments, predictions have been made as to how the system will respond to changes in process parameters, such as gas pressure, the size of reactor and if the reaction is conducted in the liquid-phase. It has been shown that natural convection is favoured by a high pressure 
in gas-phase reactions, and by a larger reactor. Because liquids have different physical properties, reactions in the liquid-phase develop more intense convection than gas-phase reactions with similar kinetic parameters.

\section{NOMENCLATURE}

\begin{tabular}{|c|c|}
\hline$a$ & concentration of intermediate $\mathrm{A}$ \\
\hline$a^{\prime}$ & dimensionless concentration of A, $a^{\prime}=a / a_{0}$ \\
\hline$a_{0}$ & scale for concentration of $\mathrm{A}$ \\
\hline$C_{P}$ & specific heat at constant pressure \\
\hline$C_{V}$ & specific heat at constant volume \\
\hline$D_{A}$ & diffusion coefficient of species A \\
\hline$E_{i}$ & activation energy of step $i$ of Sal'nikov's reaction \\
\hline$g$ & acceleration due to gravity \\
\hline$k_{i}$ & rate constant of step $i$ of the reaction \\
\hline$k_{2,0}$ & rate constant of step 2 evaluated at $T=T_{0}$ \\
\hline$L$ & characteristic length of the reactor \\
\hline$p$ & concentration of precursor $\mathrm{P}$ \\
\hline$p^{\prime}$ & dimensionless concentration of $\mathrm{P}, p^{\prime}=p / p_{0}$ \\
\hline$p_{0}$ & initial concentration of $\mathrm{P}$ \\
\hline $\mathscr{P}$ & pressure in the reactor \\
\hline$P^{\prime}$ & dimensionless pressure, $\mathscr{P}^{\prime}=\left(\mathscr{P}-\mathscr{P}_{0}\right) / \rho_{0} U^{2}$ \\
\hline $\mathscr{P}_{0}$ & initial pressure \\
\hline $\operatorname{Pr}$ & Prandtl Number, $\operatorname{Pr}=v / \kappa$ \\
\hline$q_{i}$ & exothermicity of step $i$ of the reaction \\
\hline$R$ & universal gas constant \\
\hline $\mathrm{Ra}$ & Rayleigh number, $\mathrm{Ra}=\beta g \Delta T L^{3} /(\kappa v)$ \\
\hline$t$ & time \\
\hline$t^{\prime}$ & dimensionless time, $t^{\prime}=U t / L$ \\
\hline$T$ & temperature \\
\hline$T^{\prime}$ & dimensionless temperature, $T^{\prime}=\left(T-T_{0}\right) / \Delta T$ \\
\hline$T_{0}$ & constant wall temperature \\
\hline$\underline{u}$ & velocity vector \\
\hline$\underline{\bar{u}}^{\prime}$ & dimensionless velocity vector, $\underline{u}^{\prime}=\underline{u} / U$ \\
\hline $\bar{U}$ & scale for velocity \\
\hline$\underline{x}$ & spatial coordinates \\
\hline $\bar{x}^{\prime}$ & dimensionless spatial coordinates, $\underline{x}^{\prime}=\underline{x} / L$ \\
\hline $\bar{Z}_{2}$ & pre-exponential factor in Arrhenius expression for $k_{2}$ \\
\hline$\beta$ & coefficient of thermal expansion, $\beta=1 / T$ \\
\hline$\gamma$ & ratio of specific heats \\
\hline$\Delta T$ & scale for temperature increase \\
\hline$\Delta T_{a d}$ & adiabatic temperature increase, $\Delta T_{a d}=q_{2} / C_{V}$ \\
\hline$\phi$ & dimensionless activation energy for step $2, \phi=E_{2} \Delta T / R T_{0}^{2}$ \\
\hline$\eta$ & parameter in dimensionless Arrhenius expression, $\eta=\Delta T / T_{0}$ \\
\hline
\end{tabular}




$\begin{array}{ll}\kappa & \text { thermal diffusivity } \\ v & \text { kinematic viscosity } \\ \rho & \text { density } \\ \rho_{0} & \text { density at } T=T_{0} \\ \tau_{\text {convection }} & \text { timescale for convection } \\ \tau_{\text {diffusion A }} & \text { timescale for diffusion of species A } \\ \tau_{\text {diffusion } H} & \text { timescale for diffusion of heat } \\ \tau_{\text {step } i} & \text { timescale for step } i \text { of reaction }(\mathrm{I})\end{array}$

\section{REFERENCES}

Campbell, A.N., Cardoso, S.S.S. \& Hayhurst, A.N. (2005a), “A scaling analysis of Sal'nikov's reaction, P $\rightarrow \mathrm{A} \rightarrow \mathrm{B}$, in the presence of natural convection and the diffusion of heat and matter." Proc. Roy. Soc. A. 461: $1999-2020$.

Campbell, A.N., Cardoso, S.S.S. \& Hayhurst, A.N. (2005b), "The influence of natural convection on the temporal development of the temperature and concentration fields for Sal'nikov's reaction, $\mathrm{P} \rightarrow \mathrm{A} \rightarrow \mathrm{B}$, occurring batchwise in the gas phase in a closed vessel." Chem. Eng. Sci., 60: 5705 - 5717.

Cardoso, S.S.S., Kan, P.C., Savjani, K.K., Hayhurst, A.N. \& Griffiths, J.F. (2004a), “The computation of the velocity, concentration and temperature fields during a gas-phase oscillatory reaction in a closed vessel with natural convection." Combustion and Flame, 136: 241 - 245.

Cardoso, S.S.S., Kan, P.C., Savjani, K.K., Hayhurst, A.N. \& Griffiths, J.F. (2004b), "The effect of natural convection on the gas-phase Sal'nikov reaction in a closed vessel." Physical Chemistry Chemical Physics, 6: $1687-1696$.

Fairlie, R. \& Griffiths, J.F. (2001), “A numerical study of spatial structure during oscillatory combustion in closed vessels in microgravity." Faraday Discussions, 120: 147 - 164.

Fairlie, R. \& Griffiths, J.F. (2002), "Oscillatory combustion in closed vessels under microgravity.” Mathematical and Computer Modelling, 36: 245 - 257.

Fairlie, R., Griffiths, J.F., Hughes, K.J. \& Pearlman, H. (2005) “Cool flames in space: experimental and numerical studies of propane combustion.” Proc. Combust. Inst., 30, 1057 - 1064.

Fastflo Tutorial Guide (version 3) 2000, July 1999 (online), CSIRO, Australia.

Gray, P. \& Griffiths, J.F. (1989), “Thermokinetic combustion oscillations as an alternative to thermal explosion." Combust. Flame, 78, $87-98$.

Gray, P. \& Scott, S.K. (1990a), “Chemical Oscillations and Instabilities,” Clarendon Press, Oxford, ch. 4, pp. $83-111$.

Gray, P. \& Scott, S.K. (1990b), “Chemical Oscillations and Instabilities,” Clarendon Press, Oxford, ch. 10 , pp. $264-291$. 
Griffiths, J.F., Kaye, S.R. \& Scott, S.K. (1988) "Oscillatory combustion in closed vessels: theoretical foundations and their experimental verification." $22^{\text {nd }}$ Symp. (Int.) on Combustion, The Combustion Institute, Pittsburgh, p. 1597 - 1607.

Pearlman, H. (2000), "Low-temperature oxidation reactions and cool flames at Earth and reduced gravity." Combustion and Flame, 121: 390 - 393.

Sal'nikov, I.E. (1949), "Contribution to the theory of the periodic homogeneous chemical reactions." Zhurnal Fizicheskoi Khimii, 23: 258 - 272.

Turner, J.S. (1979), “Buoyancy Effects in Fluids,” Cambridge University Press, Cambridge, ch. 7, pp. 207 -250 .

Tyler, B.J. (1966), "An experimental investigation of conductive and convective heat transfer during exothermic gas-phase reactions," Combust. Flame, 10, 90 - 91.

ACKNOWLEDGEMENTS

The financial support of the Engineering and Physical Sciences Research Council is gratefully acknowledged.

\section{Figures}

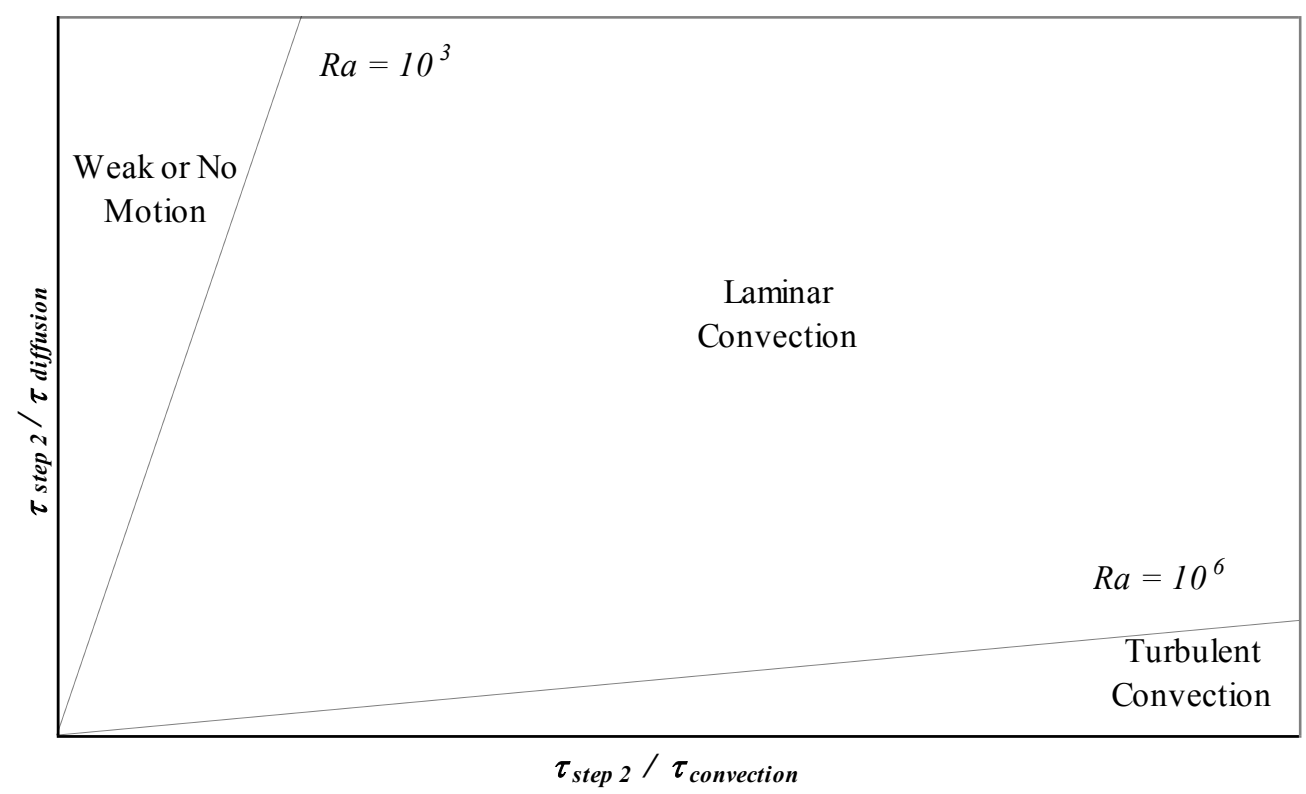

Figure 1. Regime diagram showing (for a fixed $p^{\prime}$ ) two lines of constant $\mathrm{Ra}$, and the nature of the flow in each region. 

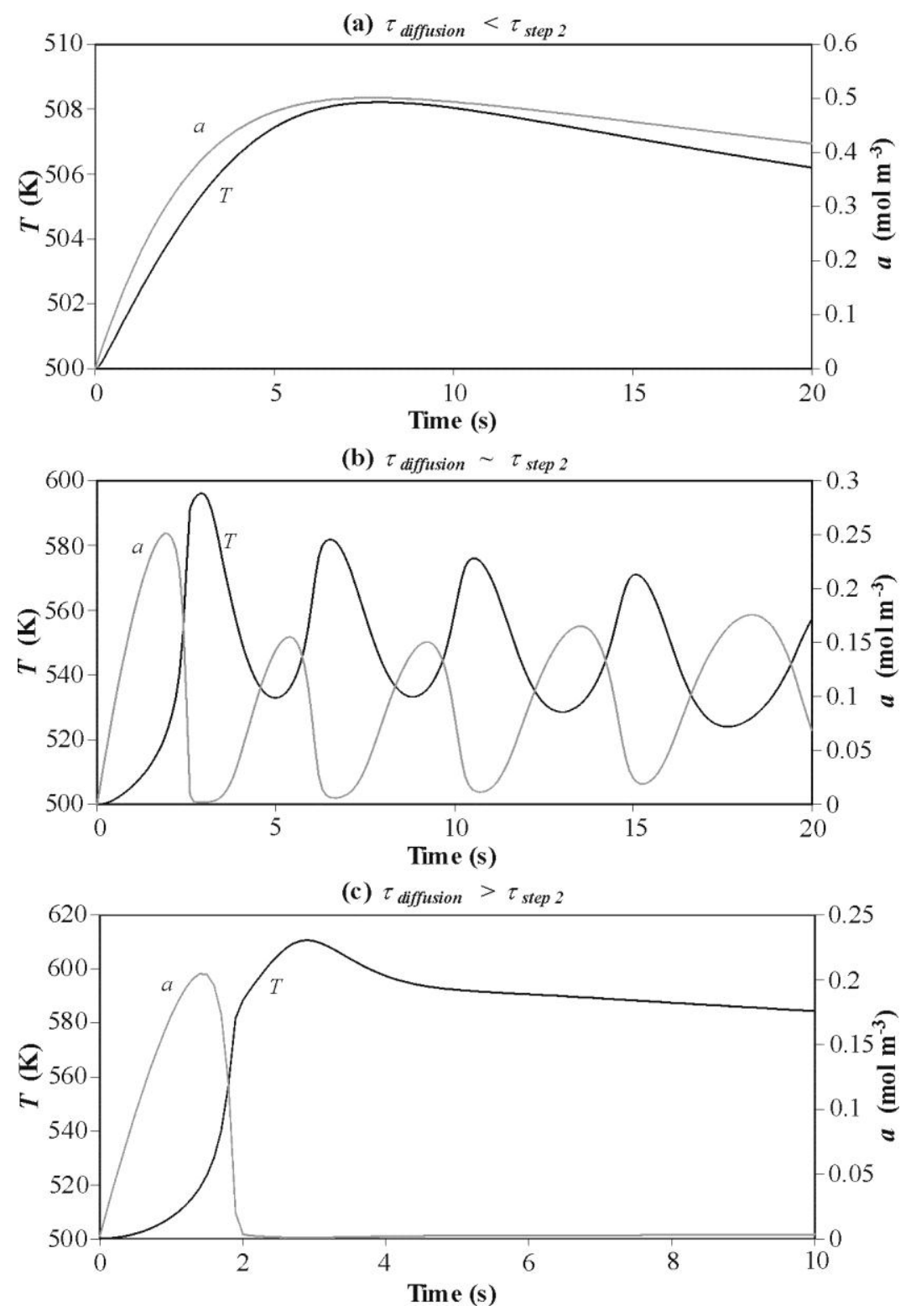

Figure 2. Plots of the temperature and concentration (a) of the intermediate $A$ at the centre of the reactor against time, when diffusion controls transport for: (a) $\tau_{\text {diffusion }}=1 \mathrm{~s} ; L=10 \mathrm{~mm}$ (b) $\tau_{\text {diffusion }}=4 \mathrm{~s} ; L=20 \mathrm{~mm}$ (c) $\tau_{\text {diffusion }}=9 \mathrm{~s} ; L=30 \mathrm{~mm}$, all for the base-case chemistry with $g=0$ $\mathrm{m} \mathrm{s}^{-2}$ and $\kappa=1 \times 10^{-4} \mathrm{~m}^{2} \mathrm{~s}^{-1}$. 


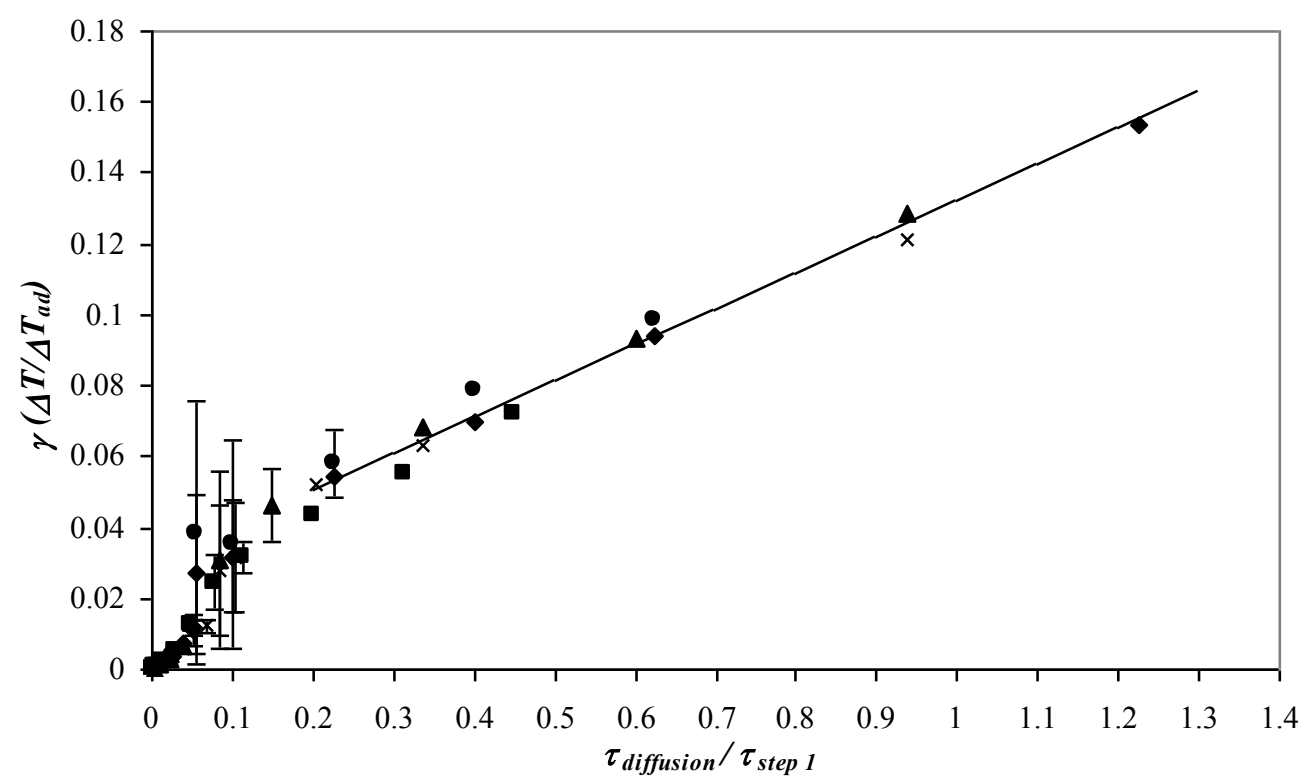

Figure 3. Plot of $\gamma\left(\Delta T / \Delta T_{a d}\right) \vee \tau_{\text {diffusion }} / \tau_{\text {step } ~}$ in the diffusive regime. The line shown corresponds to equation (18). ( $k_{l}=0.025 \mathrm{~s}^{-1}, k_{2,0}=0.265 \mathrm{~s}^{-1}, \mathrm{Ra}=0 ; \boldsymbol{\nabla} k_{l}=0.0125 \mathrm{~s}^{-1}, k_{2,0}=0.265 \mathrm{~s}^{-1}, \mathrm{Ra}=0 ; \boldsymbol{\Delta} k_{l}=$ $0.0375 \mathrm{~s}^{-1}, k_{2,0}=0.265 \mathrm{~s}^{-1}, \mathrm{Ra}=0 ; \bullet k_{l}=0.025 \mathrm{~s}^{-1}, k_{2,0}=0.132 \mathrm{~s}^{-1}, \mathrm{Ra}=0 ; \times k_{l}=0.025 \mathrm{~s}^{-1}, k_{2,0}=0.265 \mathrm{~s}^{-1}$, $\mathrm{Ra} \sim 600)$. 


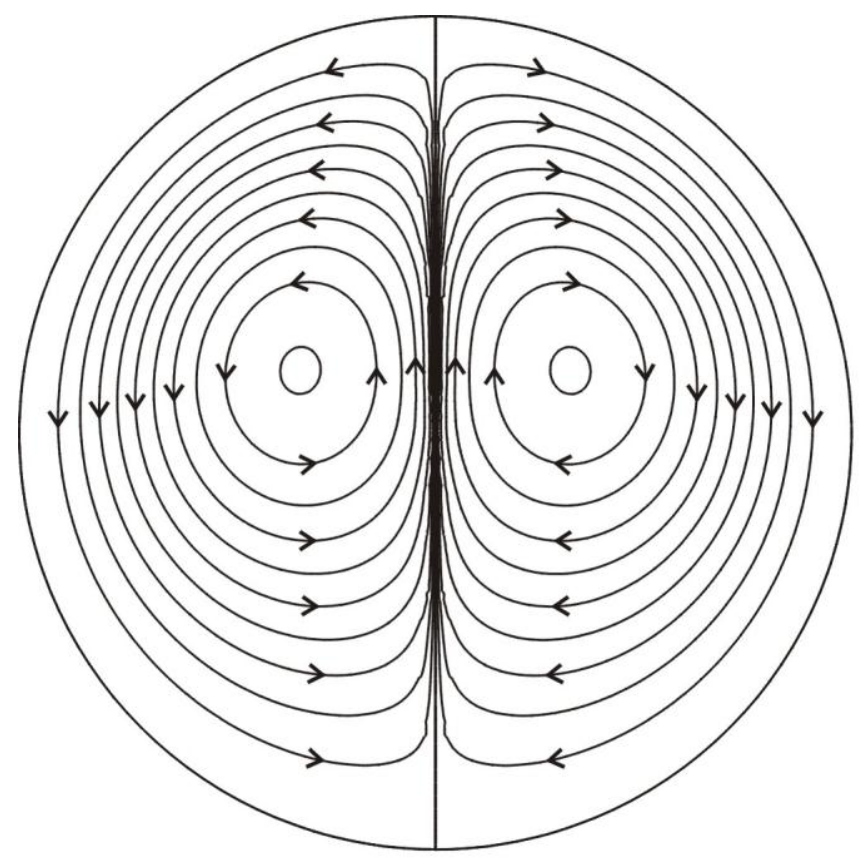

(a)

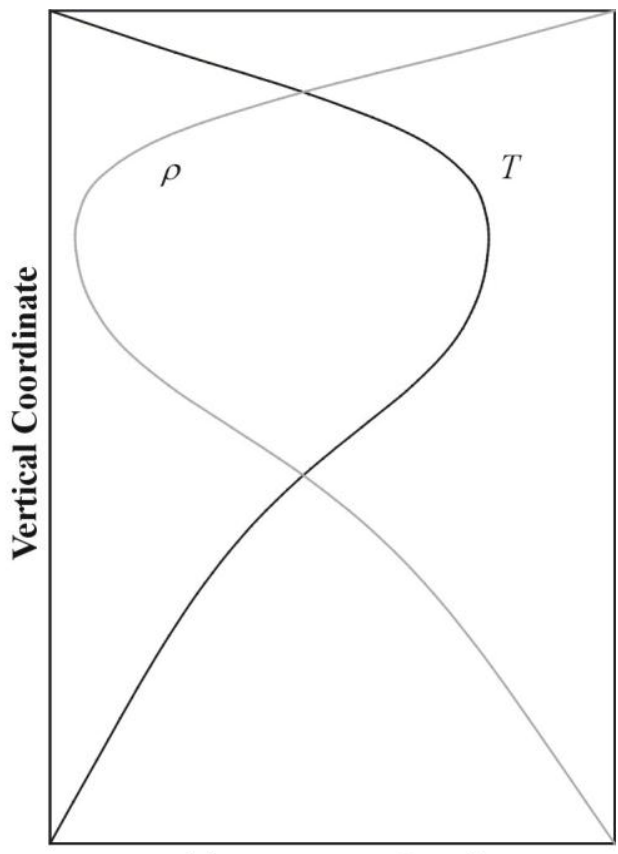

Temperature, Density

(b)

Figure 4. (a) Streamlines of the flow due to natural convection in a vertical cross section through the axis of the reactor. The toroidal vortex which typifies the flow (upwards near the axis, downwards near the wall) is shown.

(b) Temperature and density profiles along the vertical axis of the reactor, showing the unstable density distribution in the top half of the reactor, which drives flow, and the stable density distribution in the bottom half of the reactor, where flow is driven by conditions in the boundary layers. 


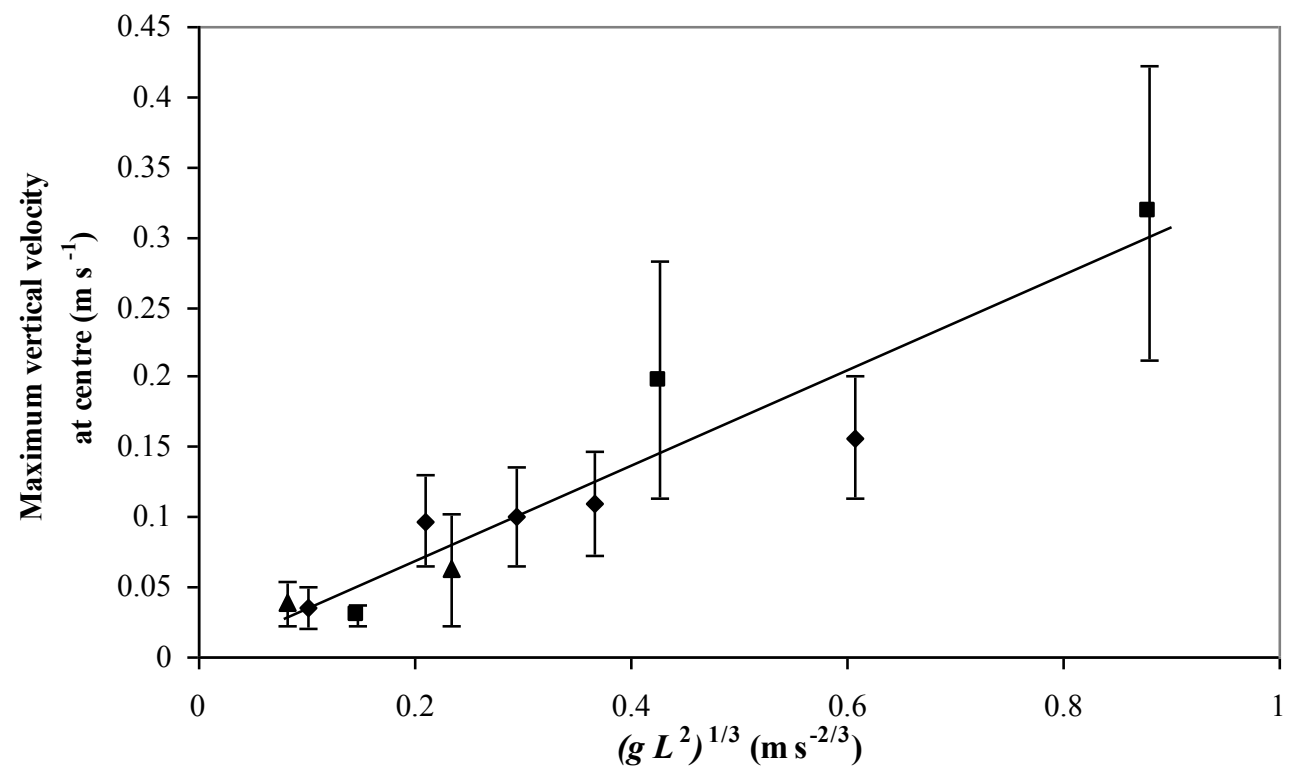

Figure 5. Plot of the maximum vertical velocity of the gas at the centre of the reactor, versus $\left(g L^{2}\right)^{1 / 3}$. The line shown corresponds to equation (19), $\mathrm{Ra} \sim 21500 .\left(-g=9.81 \mathrm{~m} \mathrm{~s}^{-2}, k_{l}=0.025 \mathrm{~s}^{-1}, k_{2,0}=0.265 \mathrm{~s}^{-1} ;\right.$ $\left.g=4.9 \mathrm{~m} \mathrm{~s}^{-2}, k_{l}=0.025 \mathrm{~s}^{-1}, k_{2,0}=0.265 \mathrm{~s}^{-1} ; \quad g=30 \mathrm{~m} \mathrm{~s}^{-2}, k_{l}=0.025 \mathrm{~s}^{-1}, k_{2,0}=0.265 \mathrm{~s}^{-1}\right)$.

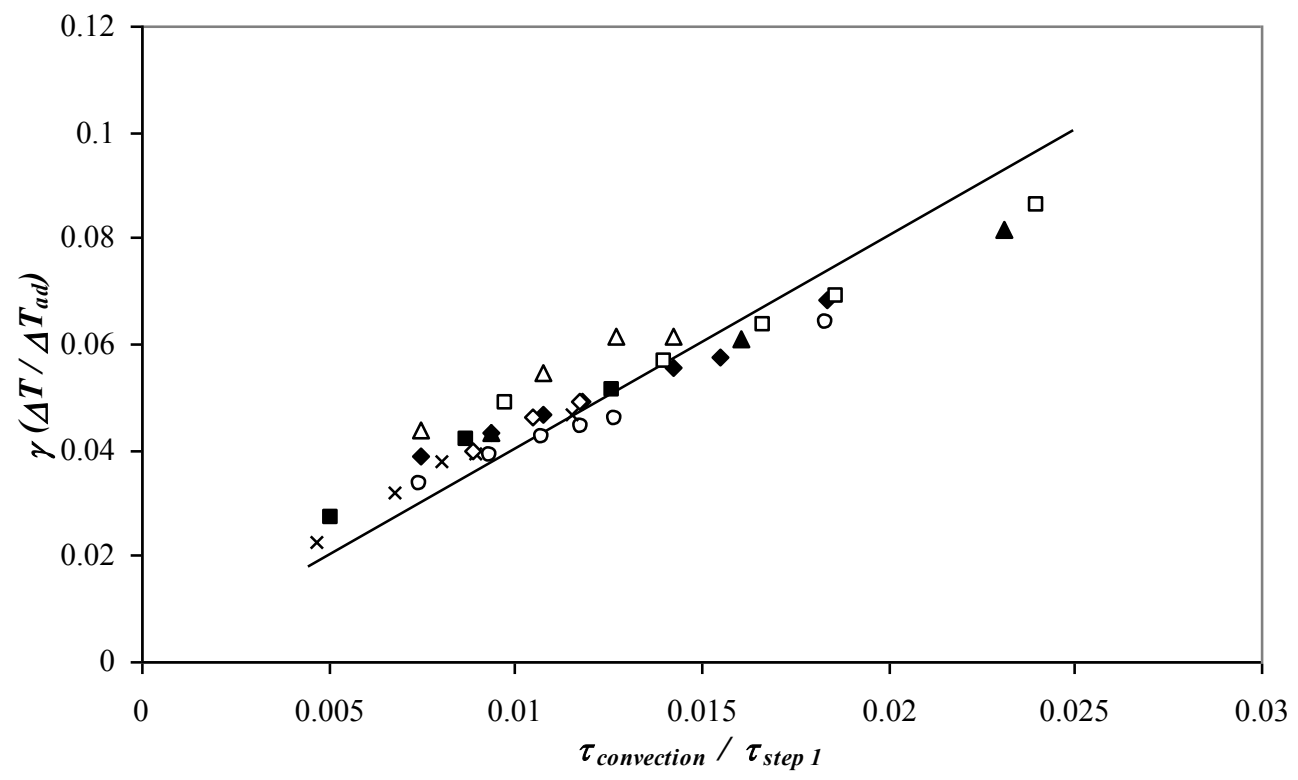

Figure 6. Plot of $\gamma\left(\Delta T / \Delta T_{a d}\right)$ versus $\tau_{\text {convection }} / \tau_{\text {step } 1}$ in the laminar convection regime. The line shown corresponds to equation (20). 'Error bars' showing the range of oscillations have been suppressed for clarity. $\left(\diamond \mathrm{g}=9.81 \mathrm{~m} \mathrm{~s}^{-2}, k_{l}=0.025 \mathrm{~s}^{-1}, k_{2,0}=0.265 \mathrm{~s}^{-1}, \mathrm{Ra} \sim 21500 ; \boldsymbol{\Delta} g=4.9 \mathrm{~m} \mathrm{~s}^{-2}, k_{l}=0.025 \mathrm{~s}^{-1}, k_{2,0}=\right.$ $0.265 \mathrm{~s}^{-1}, \mathrm{Ra} \sim 21500 ; \boldsymbol{\square} g=30 \mathrm{~m} \mathrm{~s}^{-2}, k_{l}=0.025 \mathrm{~s}^{-1}, k_{2,0}=0.265 \mathrm{~s}^{-1}, \mathrm{Ra} \sim 21500 ; \times g=9.81 \mathrm{~m} \mathrm{~s}^{-2}, k_{l}=$ $0.0125 \mathrm{~s}^{-1}, k_{2,0}=0.265 \mathrm{~s}^{-1}, \mathrm{Ra} \sim 21500 ; \diamond g=9.81 \mathrm{~m} \mathrm{~s}^{-2}, k_{l}=0.01875 \mathrm{~s}^{-1}, k_{2,0}=0.265 \mathrm{~s}^{-1}, \mathrm{Ra} \sim 21500 ; \square g$ $=9.81 \mathrm{~m} \mathrm{~s}^{-2}, k_{l}=0.0375 \mathrm{~s}^{-1}, k_{2,0}=0.265 \mathrm{~s}^{-1}, \mathrm{Ra} \sim 21500 ; \Delta g=9.81 \mathrm{~m} \mathrm{~s}^{-2}, k_{l}=0.025 \mathrm{~s}^{-1}, k_{2,0}=0.132 \mathrm{~s}^{-1}, \mathrm{Ra}$ $\left.\sim 21500 ; \mathrm{O} g=9.81 \mathrm{~m} \mathrm{~s}^{-2}, k_{l}=0.025 \mathrm{~s}^{-1}, k_{2,0}=0.265 \mathrm{~s}^{-1}, \mathrm{Ra} \sim 5000\right)$. 


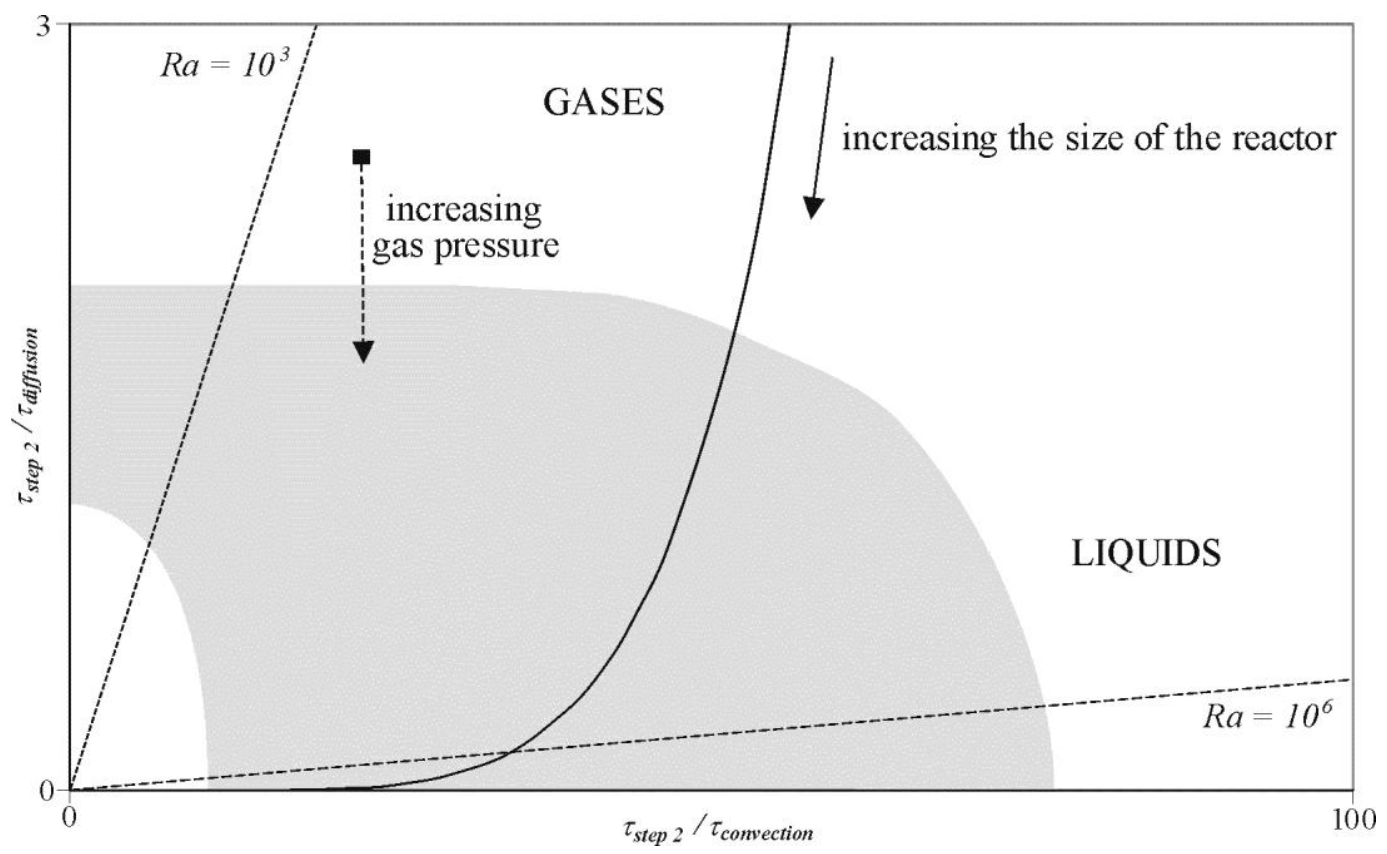

Figure 7. Regime diagram showing the effect (on the position of the working point in Fig. 1) of increasing the gas pressure, increasing the reactor's size, and the effect of performing the reaction in the liquidphase. The area in grey denotes approximately where the concentration of the intermediate and the temperature oscillate, as in Fig. $2 b$. 